\title{
LA SEMILLA ARTIFICIAL。 UNA SOLUCIÓN EN LA BIODIVERSIDAD MUNDIAL
}

\section{Orlando S. González Paneque, Juan J. Silva y Ángel Espinosa Reyes}

Centro de Estudios de Biotecnología Vegetal. Facultad de Ciencias Agrícolas. UNIVERSIDAD DE GRANMA.

Apdo. 21. Bayamo. Granma. C.P. 85100. Cuba.

E-MAIL: OGPANEQUE@UDG.CO.CU

\section{INTRODUCCIÓN}

La propagación de plantas es una ocupación básica de la humanidad y está dada en gran parte sobre la habilidad del hombre para propagar y cultivar clases específicas de plantas que puedan ser usadas como alimento, proporcionarle protección, vestido, recreo y satisfacciones estéticas (Hartmann y Kester, 1975). Se puede decir que se trata de un tipo de reproducción de plantas controlada por el hombre para perpetuar individuos escogidos o grupos de plantas que tienen para él un valor específico.

Las semillas sostienen y protegen la vida, proporcionan alimento a la humanidad, animales y otros seres vivientes, son la materia prima para la gran cantidad de productos empleados por el hombre, son riqueza y el futuro germen de una nueva generación.

La propagación "in vitro" como proceso comercial que se viene utilizando en diversas especies vegetales desde la segunda mitad del presente siglo, no ha podido emplearse con éxito en la obtención de semillas sintéticas hasta bien entrada la década de los años 80 y ha sido precisamente a lo largo de los últimos cinco años cuando se ha visto que las semillas sintéticas albergan el potencial necesario para reducir el costo de la propagación vegetal. El desarrollo de esta idea y el advenimiento de la biotecnología como nueva ciencia han abierto las puertas al desarrollo de las semillas sintéticas y ha permitido que diversas empresas y centros de investigación de todo el mundo se interesen por el tema.

Son ilimitados los campos en que se puede utilizar la biotecnología, la cual tendrá un papel tan importante como la electrónica, o tan importante como la propagación asexual, haciéndose necesario el empleo de las semillas sintéticas por las ventajas que estas ofrecen (Cantliffe et al., 1991).

Con el presente trabajo perseguimos como objetivo dar a conocer el concepto de semilla sintética o artificial y analizar las etapas principales que conducen a su obtención.

\section{DESARROLLO}

Según Boswell (1968) las semillas son una fuente de supervivencia de sus especies. Una semilla (Mckay, 1968) es un óvulo madurado que contiene un embrión. Carvalho y Nakagawa (1980) plantean 
que la semilla es un órgano de perpetuar y diseminar las especies vegetales, con capacidad de distribución y germinación en el tiempo. Las semillas generalmente están bien protegidas durante todo su desarrollo. Esta protección difiere grandemente entre las diferentes clases, tanto en grado como en la forma en que se proporciona (Boswell, 1968). Las semillas de algunas especies se desarrollan en las plantas madres con una rapidez asombrosa, otras, al contrario lo hacen sumamente despacio; ejemplo es la planta llamada pamplina que se arranca del jardín y se arroja al lado, en el momento en que sus primeras flores se abren pueden formar algunas semillas antes de que se marchiten y mueran. Las plantas más comunes forman sus semillas en un período de varios días o varias semanas después de la polinización.

Las semillas selectas son hoy uno de los más eficaces elementos para el proceso de selección en la agricultura, donde se encuentra implícito un buen grano y una buena variedad. Buen grano quiere decir semilla limpia, sana, buen tamaño, buena apariencia y germinación. Buena variedad significa perteneciente a una variedad de caracteristicas probadas de producción y calidad (Besnier, 1965).

La mayoría de las semillas sexuales se desarrollan después de la unión de las células reproductoras masculinas y femeninas; perpetuando los caracteres hereditarios constituidos por ambas células. Las plantas procedentes de semillas asexuales portan las características fenotípicas y genotípicas de la planta madre o portadora. Las plantas que reproducen fielmente su tipo por semillas de origen sexual pueden propagarse también asexualmente por medio de trozos de tallos o de otras partes apropiadas de la planta, bajo condiciones favorables, aunque estas condiciones raramente son favorables o practicables para su propagación vegetativa.

Las clases y variedades de plantas que no tienen la propiedad de producir semillas viables, es decir, que no pueden germinar y desarrollarse, deben perpetuarse por medios asexuales o como semillas artificiales.

La enorme cantidad de semillas que una sola planta de algunas especies produce, hace factible el aumento de la cantidad de semillas a una velocidad grande. Algunas otras producen pocas y el porcentaje de aumento es lento, ejemplo, una planta de tabaco puede producir hasta un millón, mientras que el chícharo de huerta, produce unas cuantas docenas a lo sumo. Muchas especies de plantas en estado de semillas conservan su poder vital por mucho tiempo, desde el momento de su formación en una estación a la siguiente, si las condiciones no le son favorables para desarrollarse; algunas lo conservan solamente un año o dos, otras por veinte años o más.

Las semillas han servido para llevar a cabo programas de mejoramiento en las plantas y obtener mayores fuentes de alimentos.

\section{HABLEMOS DE LAS SEMILAS SINTETICAS}

Las semillas sintéticas, también denominadas semillas artificiales o semillas clonales, son estructuras vegetales de origen normalmente asexual, capaces de producir un vástago (brotes y ramificaciones aéreas) y una raíz. Aademás, poseen la capacidad necesaria para regenerar una planta completamente idéntica a su progenitor (Retamal y Durán, 1989). Las estructuras vegetales a que nos referimos son los embriones somáticos y pueden obtenerse a través de las técnicas convencionales de cultivo in vitro.

Las semillas sintéticas están formadas por: embrión somático, gel y polímero.

Actualmente el proceso de fabricación de semillas sintéticas pasa por las siguientes etapas:

1. Inducción de la embriogénesis somática.

2. Obtención y selección de los embriones somáticos (ES) maduros.

3. Sincronización del crecimiento de los embriones somáticos.

4. Proliferación de ES en un biorreactor.

5. Encapsulación y recubrimiento mecánico de los ES.

Semilla sintética es un término génerico de los embriones somáticos usados en la propagación clonal de plantas (Janick et al., 1993). La tecnología de la semilla artíficial incluye dos partes: un sistema de micropropagación masiva a través del cultivo 
de tejido mediante embriones somáticos y otras por yemas (Redenbaugh, 1993).

Las semillas artificiales consisten en un embrión somático encapsulado con una cápsula de protección, que tiene el propósito de la nutrición y protección del embrión, además manifiesta un bajo costo y un alto volumen como sistema de propagación (Redenbaugh, 1990). Los avances inherentes de la semilla artificial son la producción de muchos embriones y el uso convencional de las semillas por la técnica de embriones.

\section{EL TIEMPO EN LAS SEMILLAS SINTÉTICAS}

Podemos afirmar que las semillas sintéticas apenas tienen pasado. Los antecedentes mas lejanos se remontan a la década de los años 70 cuando el profesor Murashige se dirigía a sus alumnos de la universidad de California para señalarles que “... algún día los embriones somáticos podrán ser encapsulados, convirtiéndose asi en semillas artificiales". Lo anterior está muy relacionado con el avance de la biotecnología en la esfera de la embriogénesis somática.

Desde el punto de vista científico nadie puede dudar que la primitiva idea de Murashige es hoy en día una realidad en diversos cultivos. Los países a nivel mundial que más desarrollo tienen en esta técnica resulta lógico que se encuentran entre los más desarrollados y empresas privadas que dedican gran cantidad de recursos a la obtención de semillas sintéticas. Ejemplos de estos tenemos: Japón, China, Estados Unidos, Francia, España, Canadá, entre otros. Además se trabaja en algunos países en vías de desarrollo.

Entre los cultivos en los que se ha trabajado a nivel mundial en la obtención de semillas artificiales tenemos: alfalfa, zanahoria, apio, especies forestales, cítricos, entre otros. Significativos avances se han obtenido durante los últimos diez años en la embriogénesis somática con considerable atención en la aplicación de la tecnología de la semilla artificial (Fujii et al., 1992).

Embriones somáticos de alfalfa pueden ahora ser producidos y tienen las reservas necesarias almacenadas y el vigor necesario para su conversión en el campo. Las semillas sintéticas son una revolución en el sistema de propagación en la agricultura (Fujii, et al., 1987).

El origen de la idea de una semilla artificial es difícil de determinar (Redenbaugh et al., 1990); es en los años 70 cuando comienza a emplearse el concepto como un sistema de propagación potencial para los cultivos.

La tecnología de la semilla sintética ha alcanzado avances en los últimos años, desde la primera descripción de este sistema. Puede ser usado clonalmente para la propagación de plantas empleando embriones somáticos (McKersey et al., 1989); la aplicación de esta tecnología es diversa y el desarrollo de la semilla artificial promete una seria revolución en los aspectos de micropropagación, almacenamiento de germoplasma y otros métodos de plantas de semilleros en especies importantes de cultivo.

\section{LA EMBRIOGÉNESIS SOMÁTICA}

La embriogénesis somática es la posibilidad de inducir la transformación de una célula no sexual y poco diferenciada en un grupo de células que sean capaces de originar un vástago y una raíz, constituye hoy en día, el factor limitante más importante a la hora de ampliar el número de especies en las que se pueden obtener semillas sintéticas. No obstante, la embriogénesis somática ya es una realidad y se ha obtenido en muchas especies, habiendo utilizado para ello diversas técnicas (Retamal y Durán, 1989).

Tanto desde el punto de vista morfológico como fisiológico o bioquímico las semillas sintéticas son muy similares a los verdaderos embriones de las semillas naturales; cuanto más se estudian éstos embriones somáticos más se parecen a los cigóticos, excepto lógicamente en la forma de lograr su mantenimiento y protección (Retamal y Durán, 1989).

Dada la delicada estructura que presentan los embriones somáticos, es evidente que no puedan encontrarse al descubierto; por el contrario, deben ir protegidos por un material blando y altamente hidratado que se adapte perfectamente a su estructura. Los geles son las sustancias que normalmente satisfacen todas estas necesidades, por lo tanto, las más empleadas en el recubrimiento 
en la actualidad. Un criterio importante relacionado con la semilla artificial es que pueden producirse semillas de plantas recalcitrantes en algunos cultivos tropicales, facilitándose su almacenamiento y traslado.

Los primeros requerimientos para una semilla artificial son, la obtención de embriones somáticos con resultados normales en su desarrollo. La necesidad de una alta calidad en los embriones es actualmente limitante para la comercialización de las semillas y debe de ser discutido en detalle (Redenbaugh, 1990). La envoltura que recubre el embrión debe protegerlo, facilitarle la germinación y permitir su manipulación durante su almacenamiento, transporte y plantación; debe contener nutrientes, agentes reguladores del crecimiento y otros componentes necesarios para el crecimiento y desarrollo de las plantas; por esto se hace necesario la producción del embrión para la obtención de la semilla sintética, finalmente debe considerarse que la plantación sea mecanizada.

La embriogénesis somática ofrece varios beneficios potenciales para la industria de plantas, incluyendo la proliferación en masa y la producción de metabolitos, a través del empleo de sistemas mecanizados que ofrecen posibilidades de su desarrollo (Rodríguez et al., 1990). En el caso de la zanahoria se ha empleado un método para superar el asincronismo en el desarrollo de los embriones somáticos basado en la purificación de los embriones en fase de torpedo en suspensiones de embriones heterogéneos.

La propagación del café es generalmente a través de semillas con cultivos de semilleros antes de la plantación en el campo. En el cultivo del café la propagación vegetativa fue empleada por primera vez en 1887 y estos métodos son utilizados en nuestros días (Redenbaugh, 1990).

Las semillas son normalmente producidas por plantas cultivadas como consecuencia directa de un proceso sexual (Janick et al., 1989); las semillas normales consisten en un embrión maduro con resistencia al almacenaje que presentan varios tejidos nutritivos y una envoltura necesaria para el almacenamiento de nutrientes, transporte y protección de las semillas; algo similar se persigue con la semilla artificial.

\section{ENCAPSULADO DE YEMAS}

El encapsulado puede llevarse a cabo en embriones y formar la semilla artificial, pero además pueden encapsularse yemas, lo cual tiene las ventajas siguientes: se elimina la fase de enraizamiento, aumenta el número de material de siembra, principalmente en aquellos cultivos donde la disponibilidad de semillas es limitada y especies en las que la producción de semillas botánicas es difícil, se mecaniza la fase de siembra, permite la incorporación de hormonas a la cápsula, productos fitosanitarios y microorganismos estimuladores del crecimiento, permite el almacenamiento, la conservación, la manipulación y transporte del material, favorece el intercambio de germoplasma, entre otras.

Las yemas axilares pueden ser establecidas en medios de cultivo, aisladas con una porción del tallo y mezcladas con solución de alginato de sodio. Esto se puede lograr en condiciones no estériles (semicontroladas de laboratorio), adicionándoles fungicidas para evitar la contaminación (Bapat, 1993). Las yemas encapsuladas se llevan al suelo para lograr su brotación.

\section{EMPLEO DE FERMENTADORES Y LA EXISTENCIA DE LA VARIACIÓN SOMA= CLONAL}

El análisis de la embriogénesis somática basado en el empleo de los fermentadores o biorreactores requiere de la cuantificación del desarrollo biológico, acorde con el número y la distribución por tamaño y de estructuras celulares (Harrell y Cantliffe, 1994).

Los biorreactores proporcionan muchas ventajas para el crecimiento de cultivo de plantas. La primera ventaja es el incremento en el volumen de trabajo y la segunda es que muchos biorreactores están equipados con un mecanismo que mantiene homogéneo el cultivo. Se pueden controlar el medio de cultivo y los parámetros físicos para lograr un crecimiento óptimo (Tautorus et al., 1992).

La producción de embriones somáticos en biorreactores es utilizada para la propagación masiva de plantas por cultivo de tejidos o de células. En el cultivo de células en biorreactores se producen 
metabolitos secundarios, los cuales juegan un papel importante en el empleo de las técnicas modernas (Nishimura et al., 1993). Se pueden lograr los clusters celulares aumentándose así el número de células embriogénicas aptas para la formación de embriones. La variación somaclonal puede ser detectada en los cultivos mediante variaciones genéticas, estudios fisiológicos y bioquímicos, donde estos pueden ser minimizados con el empleo de la semilla sintética (Redenbaugh, 1993b).

\section{EL COSTO DE LAS SEMILLAS SINTÉTICAS}

Según Redenbaugh (1989), citado por Retamal y Durán (1989), la posibilidad de encapsular embriones somáticos para diferentes cultivos depende fundamentalmente del costo de la estructura protectora utilizada en la encapsulación. De ahí los grandes esfuerzos que a nivel mundial los países más desarrollados están llevando a cabo para encontrar nuevos materiales, que sean más eficientes en el proceso de encapsulación o que permitan incorporar otros agentes tales como nutrientes minerales y orgánicos, reguladores del crecimiento, productos fitosanitarios e incluso microorganismos beneficiosos.

El creciente interés por la siembra directa, frente a las costosas operaciones de transplante, así como por la siembra de precisión, tanto en cultivos extensivos como en la mayor parte de los cultivos considerados tradicionalmente como hortícolas exigen cada vez más, el empleo de semillas de mayor calidad (Durán, 1989).

Según Redenbaugh (1993b), estimando el costo de la producción de la semilla sintética en el caso del cultivo de alfalfa, puede estar en los 0.068 USD por unidad y en el campo plantadas, 0.563 USD cada una; mientras que la micropropagación de las plantas ornamentales enraizadas puede estar entre 0.40 USD hasta 0.50 USD por unidad.

\section{POSIBILIDADES DEL EMPLEO DE LAS SEMILLAS SINTÉTICAS}

El gel que rodea el embrión no sólo le confiere protección sino que también lo nutre y puede controlar su crecimiento. Se pueden incorporar nutrientes minerales (nitratos, sulfatos, fosfatos, etc.), orgánicos (aminoácidos fundamentalmente), micronutrientes (boro, hierro, manganeso, molibdeno, zinc, etc.) y vitaminas, estos compuestos contribuye de forma decisiva a la nutrición de la plántula joven.

La encapsulación de embriones somáticos también permite colocar junto al embrión insecticidas, fungicidas, nematicidas $\mathrm{u}$ otros productos fitosanitarios; de este modo el embrión tendrá desde el primer momento una eficaz protección contra aquellas plagas, enfermedades $\mathrm{u}$ otros enemigos naturales de los cultivos que más puedan perjudicarle. La eficacia de este tipo de protección es mucho mayor que la utilizada de forma tradicional, ya que las cubiertas seminales pueden llegar a ser un obstáculo importante a la hora de efectuar un tratamiento fitosanitario (Retamal y Durán, 1989).

Los embriones somáticos presentan uniformidad en su crecimiento e identidad genética, por ser tejidos vegetales de la misma edad y que han sido obtenidos a partir del mismo parental y bajo las mismas condiciones de cultivo, presentando el mismo estado de desarrollo, por ende su crecimiento es totalmente sincronizado, obteniéndose finalmente en el campo cultivos más uniformes.

Aunque todavía se encuentra en fase de estudios, varias empresas de productos fitosanitarios se han interesado por la incorporación de herbicidas al gel que rodea al embrión y este aspecto ha abierto un nuevo concepto en el campo de los herbicidas. El hecho de que el gel que protege y mantiene al embrión sea una sustancia rica en agua, permite la incorporación de microorganismos, algunos de los cuales pueden favorecer el crecimiento.

Algunas plantas, como las leguminosas, permiten la incorporación de microorganismos que pueden facilitar la fijación del nitrógeno atmosférico, por ejemplo tenemos el género Rhizobium. Para controlar el crecimiento del embrión también pueden incorporarse diversos compuestos de carácter hormonal, tales como: auxinas, giberelinas y citoquininas, siendo sustancias capaces de estimular el crecimiento, o inhibidores como el ácido abscísico. De este modo es posible mantener viables los embriones durante varios días a partir de su encap- 
sulación y al mismo tiempo, estimular su capacidad de crecimiento en el momento que sean colocados en condiciones favorables de humedad, temperatura y aereación.

Las semillas sintéticas brindan la posibilidad de incorporar rápidamente cualquier logro que pueda obtenerse a partir de la manipulación genética vegetal. Según Redenbaugh (1993a), son de fácil almacenamiento, permiten obtener plantas vigorosas y uniformes en el campo, son relativamente poco costosas, contienen reservas alimenticias, fungicidas, etc., en el endospermo sintético y facilitan la siembra mecanizada.

Esta tecnología facilita el almacenamiento e intercambio de germoplasma que constituyen dos actividades de gran importancia para la agricultura (Redenbaugh, 1993b). Las semillas artificiales se obtienen a partir de embriones somáticos encapsulados, permitiéndose la propagación masal de plantas élites en invernaderos o directamente en el campo (Fujii et al., 1987).

\section{COMPORTAMIENTO EN EL CAMPO DE LAS SEMILLAS ARTIFICIALES}

Los embriones somáticos encapsulados, ofrecen un método para facilitar que el material vegetal obtenido mediante el cultivo de tejidos pueda ser sembrado directamente en invernaderos o en el campo. La propagación por semilla artificial posibilita la masividad de cultivos heterocigóticos y proporciona una alta calidad en la producción de semillas en comparación con los métodos convencionales de semilleros (Fujii et al., 1992).
Para lograr la germinación de las semillas artificiales en el suelo debe controlarse el medio ambiente, el cual es como un incubador, requerimiento este para la conversión de los embriones somáticos en raíz, vástagos y formación de hojas. Las más altas conversiones se han obtenido en alfalfa con un $20 \%$ en suelo. Por el contrario, en embriones de zanahoria con el empleo del fluido trillado en suelo de invernaderos sobreviven por espacio de 7 días pero no ocurre la conversión (Fujii et al., 1987).

\section{CONCLUSIONES}

1.- La posibilidad de obtener semillas sintéticas ha dejado de ser una ilusión científica para convertirse en una realidad e incluso en un desafío tecnológico a la hora de obtener nuevos cultivares mejor adaptados a determinados factores adversos.

2.- La automatización del proceso de obtención de semillas artificiales se impone como una necesidad para el escalado a producción.

3.- Es de vital importancia tener presente los costos para la producción de las semillas sintéticas, teniendo en cuenta que sean inferiores al de la producción de las semillas verdaderas.

\section{NOTA DE LA REDACCIÓN}

Por limitaciones de espacio no se ha podido incluir la lista completa de referencias bibliográficas que acompaña a este artículo. Los interesados en obtenerla en formato electrónico pueden solicitarla directamente a la dirección de correo electrónico de Cuadernos de Biodiversidad. 